\title{
Abdominal sacrohysteropexy versus vaginal hysterectomy for pelvic organ prolapse in young women
}

\author{
Anuja V. Bhalerao, Vaidehi A. Duddalwar*
}

Department of Obstetrics and Gynecology, N. K. P. Salve Institute of Medical Sciences, Nagpur, Maharashtra, India

Received: 11 February 2020

Accepted: 03 March 2020

*Correspondence:

Dr. Vaidehi A. Duddalwar,

E-mail: vaidehiduddalwar@gmail.com

Copyright: (C) the author(s), publisher and licensee Medip Academy. This is an open-access article distributed under the terms of the Creative Commons Attribution Non-Commercial License, which permits unrestricted non-commercial use, distribution, and reproduction in any medium, provided the original work is properly cited.

\section{ABSTRACT}

Background: Pelvic organ prolapse (POP) is the descent of the pelvic organs beyond their anatomical confines. The definitive treatment of symptomatic prolapse is surgery but its management in young is unique due to various considerations. Aim of this study was to evaluate anatomical and functional outcome after abdominal sacrohysteropexy and vaginal hysterectomy for pelvic organ prolapse in young women.

Methods: A total 27 women less than 35 years of age with pelvic organ prolapse underwent either abdominal sacrohysteropexy or vaginal hysterectomy with repair. In all women, pre-op and post-op POP-Q was done for evaluation of anatomical defect and a validated questionnaire was given for subjective outcome.

Results: Anatomical outcome was significant in both groups as per POP-Q grading but the symptomatic outcome was better for sacrohysteropexy with regard to surgical time, bleeding, ovarian conservation, urinary symptoms, sexual function.

Conclusions: Sacrohysteropexy is a better option.

Keywords: Abdominal sacrohysteropexy, Anatomical outcome, Functional outcome, Pelvic organ prolapse, Pelvic organ prolapse- quantification, Vaginal hysterectomy

\section{INTRODUCTION}

Pelvic organ prolapse (POP) has been documented in the oldest medieval literature as old as $2000 \mathrm{BC}$ with its watershed moments. Ebers Papyrus in 1500 BC described the first case. 'The judgement of surgical correction depends upon correlation of history and physical findings'- Richard Te Linde 1966. So, the definitive treatment of symptomatic prolapse or prolapse stage 2 and beyond is surgery.

Uterine prolapse is an ailment that has seemingly affected women for all the time, most of which are not treated due to lack of women coming forward with the issue. Only 11-19\% of women undergo surgery for pelvic organ prolapse. ${ }^{2-4}$ Pelvic organ prolapse is a global health problem affecting adult females of all ages. It decreases quality of life index as it increases the cost besides causing discomfort and sexual function interference considerably and it is estimated that $50 \%$ of parous women have at least minor degree of POP. ${ }^{5,6}$

Pelvic organ prolapse is generally found in peri and postmenopausal women. Obstetric trauma and multiparity are found in the etiopathogenesis of prolapse, as a result either of a direct perineal lesion or of an indirect lesion by stretching the sacral plexus. The abdominal hyper pressures of asthmatics and family history are also described as risk factors for the occurrence of urogenital prolapse. ${ }^{7,8}$ But in young women it is a rare phenomenon with a clinical background and postulation as given by Jackson in 1996. Mechanical stability of urogenital tract depends on intact collagen fibres. Newly formed 
immature collagen is degraded easily along with increased metalloproteinase activity making the collagen brittle and susceptible to rupture. Conventionally known environmental factors such as age or obstetric trauma are no longer sufficient to explain the genesis and progression of prolapse, especially in young women. The identification of risk factors is not limited to epidemiological but also therapeutic interest. Loss of integrity of uterosacral and cardinal ligament complex leads to weakening of pelvic organ support. Prolapse is therefore, a consequence of deficiency rather than precipitating factors causing prolapse..$^{9-11}$

Contemporary understanding of the dynamic anatomy of pelvic floor support has led us to new conservative surgery for the management of uterine prolapse. The uterus itself does not play any role in the pathogenesis of uterine prolapse. Therefore, hysterectomy should not be the prime treatment and fixing of cervix to strong ligaments such as sacrospinous or anterior longitudinal ligament could give a more successful result leading to conservation of uterus in young women. ${ }^{12}$

Exact etiological data is not known and there is paucity of Cochrane data regarding recommendations of surgeries. Also, apical prolapse in young has concomitant stress urinary incontinence. Recurrences are more common in young women compared to old and choice of surgery thus becomes a dilemma and puts the surgeon in a quandry. Therefore, treatment of genital prolapse in young women is unexplored, intuitive and has many controversies today. Uterine conservation has ushered enormously in the era of technological upgradation and improvement in sutures considering patient preference and satisfaction. So, although anatomical outcome of abdominal sacrohysteropexy appears to be good, measurement of functional outcome both pre and postoperatively is necessary for future recommendations of this surgery and hence the study has been undertaken. Moreover, reconstructive surgeons are inspired and fascinated due to intricacies and challenges and hence genital prolapse in young women is a topical issue.

\section{Research question}

Is abdominal sacrohysteropexy better choice than vaginal hysterectomy with pelvic floor repair in young women with pelvic organ prolapse?

\section{METHODS}

This hospital based prospective and comparative study was conducted in the department of obstetrics and gynecology at N. K. P. Salve Institute of Medical Sciences, Nagpur, Maharashtra over a period of 18 months from $1^{\text {st }}$ January 2017 to $30^{\text {th }}$ June 2018. 27 women less than 35 years of age with symptomatic prolapse, requiring surgery, willing to participate in the study were assessed and enrolled in the study as per formulated inclusion and exclusion criteria.
Convenient sampling method was incorporated. All women attending gynecology OPD having symptoms of mass coming out per vaginum, willing for surgical correction and non-compliant to conservative management were included in the study. Women with abnormal uterus and ovaries on ultrasound, abnormal bleeding patterns and abnormal cervical cytology were excluded from the study. Women were then subjected to standardized questionnaire including their demographic details, detailed history of chief complaints (onset and duration of symptoms, progression, aggravating and relieving factors, reducibility of mass) age at marriage, detailed menstrual, obstetric and past histories with associated urogenital and defecatory symptoms. History of medical comorbidities and precipitating factors of prolapse were noted. Family and personal histories were taken into consideration. Thorough general, systemic, spine and local examination was done. Pelvic examination was done for assessing grade, level and complexity of prolapse. Associated SUI was noted. The selected cohort of women underwent assessment by pelvic organ prolapse quantification (POP-Q) system preoperatively. All required investigations and prerequisites of surgeries were performed, following which women were subjected either to abdominal sacrohysteropexy or vaginal hysterectomy after convenient sampling and random allocation. Abdominal sacrohysteropexy was performed in 12 women while vaginal hysterectomy with pelvic floor repair was performed in 15 women.

All the surgeries were performed by a single surgeon to avoid observational bias. Simultaneously intra-op complications were noted and post-op quantification of prolapse was done by POP-Q system. Subsequently, postop assessment by POP-Q was also done at 1,3, and 6 months in all women when they came for follow-up. The anatomical success of surgery was assessed depending upon the decrease in the stage $<2$ as per POP-Q and functional success was assessed by a validated questionnaire to determine the relief of symptoms and quality of life on the basis of reduction of bulge symptoms and dyspareunia.

\section{Statistical analysis}

All findings were noted and statistical analysis was done by application of Chi square test, Fisher exact test and test for estimation of null hypothesis, subsequent to which, comparison was done to determine the anatomical and functional outcome in young women with pelvic organ prolapse after abdominal sacrohysteropexy or vaginal hysterectomy. p- value $<0.05$ was considered significant

\section{RESULTS}

\section{Distribution of women according to age}

Maximum women who underwent abdominal sacrohysteropexy belonged to the age group of 21-25 
years and those who underwent vaginal hysterectomy belonged to $31-35$ years.

Mean age for the patients who underwent abdominal sacrohysteropexy was 25.6 years and for those who underwent vaginal hysterectomy was 31.9 years (Table 1).

\section{Distribution of women according to parity}

5 women with parity of 1-2 underwent abdominal sacrohysteropexy while 7 women with parity of 1-2 and $>2$ underwent vaginal hysterectomy.

Mean parity for abdominal sacrohysteropexy was $33.34 \%$ among nullipara and $66.67 \%$ among multipara. Mean parity for vaginal hysterectomy was $6.67 \%$ among nullipara and $93.34 \%$ among multipara (Table 2).

\section{Distribution of women according to degree of prolapse}

$91.66 \%$ patients who underwent abdominal sacrohysteropexy and $80 \%$ of them who underwent vaginal hysterectomy had $3^{\text {rd }}$ degree prolapse. Procidentia was seen in $8.33 \%$ woman who underwent abdominal sacrohysteropexy and $20 \%$ women who underwent vaginal hysterectomy (Table 3 ).

\section{Distribution of women according to Level of vaginal wall prolapse}

Most of the patients who underwent abdominal sacrohysteropexy or vaginal hysterectomy had 1 st and 2 nd level of prolapse. All 3 levels of prolapse was seen in 1 woman from each group. Level 1 prolapse was seen in 1 and 4 women who underwent abdominal sacrohysteropexy and vaginal hysterectomy respectively (Table 4).

Table 1: Distribution of women according to age.

\begin{tabular}{|c|c|c|c|c|}
\hline & & & Abdominal sacrohysteropexy & Vaginal hysterectomy \\
\hline \multirow{6}{*}{ Age } & \multirow{2}{*}{$21-25$} & Count & 6 & 0 \\
\hline & & $\%$ within age & $50.0 \%$ & $0.0 \%$ \\
\hline & \multirow{2}{*}{$26-30$} & Count & 4 & 2 \\
\hline & & $\%$ within age & $33.3 \%$ & $13.33 \%$ \\
\hline & \multirow{2}{*}{$31-35$} & Count & 2 & 13 \\
\hline & & $\%$ within age & $16.66 \%$ & $86.7 \%$ \\
\hline Total & & Count & 12 & 15 \\
\hline
\end{tabular}

Table 2: Distribution of women according to parity.

\begin{tabular}{|c|c|c|c|c|}
\hline & & & Abdominal sacrohysteropexy & Vaginal hysterectomy \\
\hline \multirow{6}{*}{ Parity } & \multirow{2}{*}{ Nulli } & Count & 4 & 1 \\
\hline & & $\%$ within parity & $33 \%$ & $6.66 \%$ \\
\hline & \multirow{2}{*}{$1-2$} & Count & 5 & 7 \\
\hline & & $\%$ within parity & $41.7 \%$ & $46.66 \%$ \\
\hline & \multirow{2}{*}{$>2$} & Count & 3 & 7 \\
\hline & & $\%$ within parity & $25 \%$ & $46.66 \%$ \\
\hline Total & & Count & 12 & 15 \\
\hline
\end{tabular}

Table 3: Distribution of women according to degree of prolapse.

\begin{tabular}{|c|c|c|c|c|}
\hline & & & Abdominal sacrohysteropexy & Vaginal hysterectomy \\
\hline \multirow{4}{*}{$\begin{array}{l}\text { Degree } \\
1,2,3,4\end{array}$} & \multirow{2}{*}{3} & Count & 11 & 12 \\
\hline & & $\%$ within degree $1,2,3,4$ & $91.66 \%$ & $80 \%$ \\
\hline & \multirow{2}{*}{4} & Count & 1 & 3 \\
\hline & & $\%$ within degree $1,2,3,4$ & $8.33 \%$ & $20 \%$ \\
\hline Total & & Count & 12 & 15 \\
\hline
\end{tabular}

Table 4: Distribution of women according to level of vaginal wall prolapse.

\begin{tabular}{|c|c|c|c|c|}
\hline & & Abdominal sacrohysteropexy & Vaginal hysterectomy & Total \\
\hline \multirow{3}{*}{$\begin{array}{l}\text { Level of vaginal } \\
\text { wall prolapse }\end{array}$} & 1,2 & 10 & 10 & 20 \\
\hline & $1,2,3$ & 1 & 1 & 2 \\
\hline & 1 & 1 & 4 & 5 \\
\hline \multicolumn{2}{|l|}{ Total } & 12 & 15 & 27 \\
\hline
\end{tabular}


Table 5: Comparison of mean pre-operative and post-operative POP-Q of women (abdominal sacrohysteropexy).

\begin{tabular}{|c|c|c|c|c|c|c|}
\hline \multicolumn{7}{|c|}{ Paired samples statistics (abdominal sacrohysteropexy) } \\
\hline & & Mean & SD & Std. error mean & $\mathbf{t}$ & $\mathbf{p}$ \\
\hline \multirow{2}{*}{ Pair 1} & POPQ pre-op in cms Aa & 0.08 & 0.515 & 0.149 & \multirow{2}{*}{6.141} & \multirow{2}{*}{$<0.001$} \\
\hline & POPQ post-op anatomical Aa & -1.92 & 0.996 & 0.288 & & \\
\hline \multirow{2}{*}{ Pair 2} & POPQ pre-op in cms $\mathrm{Ba}$ & 0.08 & 0.515 & 0.149 & \multirow{2}{*}{6.092} & \multirow{2}{*}{$<0.001$} \\
\hline & POPQ post-op anatomical $\mathrm{Ba}$ & -1.50 & 0.905 & 0.261 & & \\
\hline \multirow{2}{*}{ Pair 3} & POPQ pre-op in cms C & 5.50 & 1.000 & 0.289 & \multirow{2}{*}{15.014} & \multirow{2}{*}{$<0.001$} \\
\hline & POPQ post-op anatomical C & -2.83 & 1.586 & 0.458 & & \\
\hline \multirow{2}{*}{ Pair 4} & POPQ pre-op in cms Ap & -0.92 & 0.289 & 0.083 & \multirow{2}{*}{4.733} & \multirow{2}{*}{0.001} \\
\hline & POPQ post-op anatomical Ap & -2.00 & 0.739 & 0.213 & & \\
\hline \multirow{2}{*}{ Pair 5} & POPQ pre-op in $\mathrm{cms} \mathrm{Bp}$ & -1.75 & 0.866 & 0.250 & \multirow{2}{*}{-1.301} & \multirow{2}{*}{0.220} \\
\hline & POPQ post-op anatomical Bp & -1.42 & 0.515 & 0.149 & & \\
\hline \multirow{2}{*}{ Pair 6} & POPQ pre-op in cms D & -2.42 & 1.505 & 0.434 & \multirow{2}{*}{10.352} & \multirow{2}{*}{$<0.001$} \\
\hline & POPQ post-op anatomical D & -6.67 & 0.778 & 0.225 & & \\
\hline \multirow{2}{*}{ Pair 7} & POPQ pre-op in cms TVL & 8.08 & 0.289 & 0.083 & \multirow{2}{*}{-0.209} & \multirow{2}{*}{0.838} \\
\hline & POPQ post-op anatomical TVL & 8.17 & 1.403 & 0.405 & & \\
\hline \multirow{2}{*}{ Pair 8} & POPQ pre-op in cms PB & 3.67 & 0.492 & 0.142 & \multirow{2}{*}{-0.561} & \multirow{2}{*}{0.586} \\
\hline & POPQ post-op anatomical PB & 3.75 & 0.452 & 0.131 & & \\
\hline \multirow{2}{*}{ Pair 9} & POPQ pre-op in cms Gh & 4.33 & 0.492 & 0.142 & \multirow{2}{*}{-3.023} & \multirow{2}{*}{0.012} \\
\hline & POPQ post-op anatomical Gh & 4.92 & 0.289 & 0.083 & & \\
\hline
\end{tabular}

Table 6: Comparison of mean pre-operative and post-operative POP-Q of women (vaginal hysterectomy).

\begin{tabular}{|c|c|c|c|c|c|c|}
\hline & & Mean & SD & Std. error mean & $\mathbf{t}$ & p \\
\hline \multirow{2}{*}{ Pair 1} & POPQ preop in cms Aa & 0.93 & 0.961 & 0.248 & \multirow{2}{*}{10.986} & \multirow{2}{*}{$<0.001$} \\
\hline & POPQ postop anatomical Aa & -2.40 & 0.507 & 0.131 & & \\
\hline \multirow{2}{*}{ Pair 2} & POPQ preop in $\mathrm{cms} \mathrm{Ba}$ & 0.93 & 0.961 & 0.248 & \multirow{2}{*}{8.290} & \multirow{2}{*}{$<0.001$} \\
\hline & POPQ postop anatomical $\mathrm{Ba}$ & -1.47 & 0.516 & 0.133 & & \\
\hline \multirow{2}{*}{ Pair 3} & POPQ preop in cms C & 3.73 & 1.438 & 0.371 & \multirow{2}{*}{18.491} & \multirow{2}{*}{$<0.001$} \\
\hline & POPQ postop anatomical $\mathrm{C}$ & -3.07 & 0.594 & 0.153 & & \\
\hline \multirow{2}{*}{ Pair 4} & POPQ preop in $\mathrm{cms} A p$ & -0.13 & 1.506 & 0.389 & \multirow{2}{*}{4.571} & \multirow{2}{*}{$<0.001$} \\
\hline & POPQ postop anatomical Ap & -2.20 & 0.775 & 0.200 & & \\
\hline \multirow{2}{*}{ Pair 5} & POPQ preop in $\mathrm{cms} \mathrm{Bp}$ & 0.00 & 2.070 & 0.535 & \multirow{2}{*}{2.505} & \multirow{2}{*}{0.025} \\
\hline & POPQ postop anatomical Bp & -1.40 & 0.507 & 0.131 & & \\
\hline \multirow{2}{*}{ Pair 6} & POPQ preop in cms D & -3.53 & 1.767 & 0.456 & \multirow{2}{*}{5.469} & \multirow{2}{*}{$<0.001$} \\
\hline & POPQ postop anatomical D & -6.80 & 1.014 & 0.262 & & \\
\hline \multirow{2}{*}{ Pair 7} & POPQ preop in cms TVL & 8.07 & 0.704 & 0.182 & \multirow{2}{*}{-3.228} & \multirow{2}{*}{0.006} \\
\hline & POPQ postop anatomical TVL & 8.60 & 0.507 & 0.131 & & \\
\hline \multirow{2}{*}{ Pair 8} & POPQ preop in cms PB & 3.47 & 0.834 & 0.215 & \multirow{2}{*}{-0.899} & \multirow{2}{*}{0.384} \\
\hline & POPQ postop anatomical PB & 3.67 & 0.488 & 0.126 & & \\
\hline \multirow{2}{*}{ Pair 9} & POPQ preop in $\mathrm{cms}$ Gh & 4.60 & 0.507 & 0.131 & \multirow{2}{*}{-3.055} & \multirow{2}{*}{0.009} \\
\hline & POPQ postop anatomical Gh & 5.00 & 0.000 & 0.000 & & \\
\hline
\end{tabular}

\section{Comparison of mean pre-operative and post-operative} $P O P-Q$ of women (abdominal sacrohysteropexy)

Pre-operative and post-operative evaluation of women who underwent abdominal sacrohysteropexy by POP-Q system shows that the $\mathrm{p}$-value of all the parameters of POP-Q system was significant except Bp (most dependent portion on posterior vaginal wall, between Ap and posterior fornix), TVL (total vaginal length) and PB (perineal body). This indicates that all the parameters of POP-Q were significantly reduced post-operatively amongst women who underwent abdominal sacrohysteropexy except total vaginal length, perineal body and most dependant point on posterior vaginal wall even with the desired anatomical changes (Table 5).

Comparison of mean pre-operative and post-operative $P O P-Q$ of women (vaginal hysterectomy)

Pre-op and post-op evaluation of women who underwent vaginal hysterectomy by POP-Q system shows that the $\mathrm{p}$ - 
value of all the parameters of POP-Q system was significant except PB (perineal body).

This shows that amongst the women who underwent vaginal hysterectomy all the parameters of POP-Q were significantly reduced post-surgery except perineal body (Table 6).
Comparison of post-op POP-Q of women (abdominal sacrohysteropexy and vaginal hysterectomy)

Comparison of post-operative findings of women who underwent abdominal sacrohysteropexy and vaginal hysterectomy by POP-Q assessment system shows that the $\mathrm{p}$-value of all 9 parameters of post-operative POP-Q were not statistically significant.

Table 7: Comparison of post-operative POP-Q of women (abdominal sacrohysteropexy and vaginal hysterectomy).

\begin{tabular}{|c|c|c|c|c|c|c|c|}
\hline \multicolumn{8}{|l|}{ Group statistics } \\
\hline & Group & $\mathbf{N}$ & Mean & SD & Std. error mean & $\mathbf{t}$ & p \\
\hline \multirow{2}{*}{ POPQ postop anatomical Aa } & AS & 12 & -2.92 & 0.996 & 0.288 & \multirow{2}{*}{-1.76} & \multirow{2}{*}{0.09} \\
\hline & $\mathrm{VH}$ & 15 & -2.40 & 0.507 & 0.131 & & \\
\hline \multirow{2}{*}{ POPQ postop anatomical $\mathrm{Ba}$} & AS & 12 & -1.50 & 0.905 & 0.261 & \multirow{2}{*}{-0.108} & \multirow{2}{*}{0.914} \\
\hline & $\mathrm{VH}$ & 15 & -1.47 & 0.516 & 0.133 & & \\
\hline \multirow{2}{*}{ POPQ postop anatomical $\mathrm{C}$} & AS & 12 & -3.83 & 1.586 & 0.458 & \multirow{2}{*}{-1.718} & \multirow{2}{*}{0.098} \\
\hline & VH & 15 & -3.07 & 0.594 & 0.153 & & \\
\hline \multirow{2}{*}{ POPQ postop anatomical Ap } & AS & 12 & -2.50 & 0.739 & 0.213 & \multirow{2}{*}{1.02} & \multirow{2}{*}{0.317} \\
\hline & $\mathrm{VH}$ & 15 & -2.20 & 0.775 & 0.200 & & \\
\hline \multirow{2}{*}{ POPQ postop anatomical Bp } & AS & 12 & -1.42 & 0.515 & 0.149 & \multirow{2}{*}{-0.101} & \multirow{2}{*}{0.92} \\
\hline & $\mathrm{VH}$ & 15 & -1.40 & 0.507 & 0.131 & & \\
\hline \multirow{2}{*}{ POPQ postop anatomical D } & AS & 12 & -6.64 & 0.809 & 0.244 & \multirow{2}{*}{0.722} & \multirow{2}{*}{0.476} \\
\hline & VH & 15 & -6.80 & 1.014 & 0.262 & & \\
\hline \multirow{2}{*}{ POPQ postop anatomical TVL } & AS & 12 & 8.17 & 1.403 & 0.405 & \multirow{2}{*}{-1.68} & \multirow{2}{*}{0.1 .3} \\
\hline & VH & 15 & 8.60 & 0.507 & 0.131 & & \\
\hline \multirow{2}{*}{ POPQ postop anatomical PB } & AS & 12 & 3.75 & 0.452 & 0.131 & \multirow{2}{*}{0.437} & \multirow{2}{*}{0.665} \\
\hline & VH & 15 & 3.67 & 0.488 & 0.126 & & \\
\hline \multirow{2}{*}{ POPQ postop anatomical Gh } & AS & 12 & 4.92 & 0.289 & 0.083 & \multirow{2}{*}{-1.07} & \multirow{2}{*}{0.295} \\
\hline & VH & 15 & 5.00 & 0.000 & 0.000 & & \\
\hline
\end{tabular}

Table 8: Distribution of women according to complications of surgery.

\begin{tabular}{|llll|l|}
\hline & & & Abdominal sacrohysteropexy & Vaginal hysterectomy \\
\hline \multirow{3}{*}{ Complications } & \multirow{2}{*}{ Haemorrhage } & Count & 0 & 4 \\
\cline { 2 - 5 } & \multirow{2}{*}{ Nil } & \% within complications & $0.0 \%$ & $26.66 \%$ \\
\hline \multirow{2}{*}{ Total } & Count & 12 & 11 \\
\cline { 2 - 5 } & & \% within complications & $100 \%$ & $73.33 \%$ \\
\hline
\end{tabular}

Table 9: Distribution of women according to risk factors of prolapse.

\begin{tabular}{|c|c|c|c|c|}
\hline & & & $\begin{array}{l}\text { Abdominal } \\
\text { sacrohysteropexy }\end{array}$ & $\begin{array}{l}\text { Vaginal } \\
\text { hysterectomy }\end{array}$ \\
\hline \multirow{4}{*}{$\begin{array}{l}\text { Risk } \\
\text { factor - } \\
\text { family } \\
\text { history }\end{array}$} & \multirow{2}{*}{$\begin{array}{l}\text { Family } \\
\text { history }\end{array}$} & Count & 7 & 2 \\
\hline & & $\%$ within risk factor for recurrence, family history & $58.33 \%$ & $13.33 \%$ \\
\hline & \multirow{2}{*}{ None } & Count & 5 & 13 \\
\hline & & $\%$ within risk factor for recurrence, family history & $41.66 \%$ & $86.66 \%$ \\
\hline Total & & Count & 12 & 15 \\
\hline
\end{tabular}

Table 10: Comparison of women according to anatomical and functional outcome after surgery.

\begin{tabular}{|l|l|l|}
\hline Surgery & Abdominal sacrohysteropexy & Vaginal hysterectomy \\
\hline Functional outcome & $11 / 12(91.67 \%)$ & $12 / 15(80 \%)$ \\
\hline Anatomical outcome & $100 \%$ & $100 \%$ \\
\hline
\end{tabular}


Thereby, concluding that the post-operative findings of women after abdominal sacrohysteropexy were comparable with the post-operative findings of women who underwent vaginal hysterectomy (Table 7).

\section{Distribution of women according to complications of surgery}

No intra-operative or post-operative complications were encountered in women who underwent abdominal sacrohysteropexy. $4(26.66 \%)$ women who underwent vaginal hysterectomy had post-operative complication of haemorrhage (Table 8).

\section{Distribution of women according to risk factors of prolapse}

$58.33 \%$ patients who underwent abdominal sacrohysteropexy and $13.33 \%$ patients who underwent vaginal hysterectomy had a positive family history for pelvic organ prolapse. Risk factors for pelvic organ prolapse were not encountered in rest of the women in either groups (Table 9).

Comparison of women according to anatomical and functional outcome after surgery

A total $91.67 \%$ women who underwent abdominal sacrohysteropexy had functional improvement compared to $80 \%$ women who underwent vaginal hysterectomy.
Anatomical outcome in both the groups was same (100\%) (Table 10)

\section{DISCUSSION}

Pelvic organ prolapse occurs as a result of disturbance of anatomical and mechanical equilibrium. The normal position, support and suspension of uterus with other pelvic organs rely on an interdependent system of bony, muscular and connective tissue elements. This threedimensional system, if presents with subtle variation in one part may lead to stresses in other part that eventually lead to alteration or failure of normal anatomy. In theory, if one of these elements fail, the others can supply it within certain limits. Pelvic organ prolapse in young women is rare. The prevalence increases with age and remains stable later on. Common etiological factors leading to prolapse in young women are: vaginal births, obesity, family history, race and ethnicity, alteration in collagen content of various tissues and abnormal collagen synthesis.

The primary aim of surgery is to relieve symptoms, which may be caused by prolapse, and in most cases to restore vaginal anatomy so that sexual function may be maintained or improved without significant adverse effects or complications. The type of operation to be employed depends upon various factors - age, parity, degree and type of prolapse, desirous of further child bearing, desirous of menstrual and sexual function, general physical condition of the patient.

Table 11: Comparison with other studies.

\begin{tabular}{|c|c|c|c|c|c|c|c|}
\hline Study & $\begin{array}{l}\text { Mean } \\
\text { age }\end{array}$ & Mean parity & $\begin{array}{l}\text { Grade of } \\
\text { prolapse }\end{array}$ & $\begin{array}{l}\text { Success } \\
\text { rate }\end{array}$ & $\begin{array}{l}\text { Recurrence } \\
\text { rate }\end{array}$ & Complications & $\begin{array}{l}\text { Follow- } \\
\text { up }\end{array}$ \\
\hline Tahir $^{13}(12)$ & 30 years & $\begin{array}{l}83.3 \% \text { multi } \\
16.6 \% \text { nulli }\end{array}$ & II & $83.3 \%$ & $16.6 \%$ & - & $\begin{array}{l}12 \\
\text { months }\end{array}$ \\
\hline Barranger $^{14}(30)$ & 35.7 years & 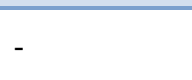 & II / III & $93.4 \%$ & $6.6 \%$ & $\begin{array}{l}1 \text { mesh rupture } \\
4 \text { intra-op }\end{array}$ & $\begin{array}{l}44.5 \\
\text { months }\end{array}$ \\
\hline Demirci and leron ${ }^{15}(20)$ & - & - & III & $95 \%$ & $5 \%$ & 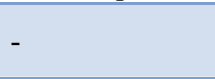 & $\begin{array}{l}25 \\
\text { months }\end{array}$ \\
\hline This study (12) & 25.6 years & $\begin{array}{l}66.67 \% \text { multi } \\
33.34 \% \text { nulli }\end{array}$ & III & $100 \%$ & - & - & $\begin{array}{l}6-7 \\
\text { months }\end{array}$ \\
\hline
\end{tabular}

The two procedures - abdominal sacrohysteropexy and vaginal hysterectomy are comparable with respect to complication rate, recurrence rate, quality of life and functional outcome at 6-7 months of follow-up. 27 young women were selected for this study and we aimed to see the results of uterine preservation in them.

The conventional surgical treatment for POP is vaginal hysterectomy with pelvic floor repair. Uterus is removed despite being normal. Moreover, the removal of uterus fails to address the etiology of prolapse. Literature states $40 \%$ of women experienced vaginal vault prolapse and there was two-fold increase in risk of ovarian function failure subsequent to vaginal hysterectomy.
Young women desire for uterine preservation with increasing awareness. Uterine conservation not only supports the pelvic floor, it preserves fertility, improves sexual function and wellbeing and decreases the various risks associated with hysterectomy - reduced operation time, estimated blood loss and post-op recovery time. ${ }^{12}$ So, non-surgical modalities remain the first line of management of prolapse. Amongst uterine preservation surgeries, abdominal sacrohysteropexy is most widely used.

Abdominal sacrohysteropexy is a surgical procedure to correct uterine prolapse. It involves resuspension of the prolapsed uterus using a strip of synthetic mesh to lift the 
uterus and hold it in place via abdominal approach. Sacrohysteropexy results in satisfactory anatomy and functional result with normal vaginal axis. Disadvantages of abdominal sacrohysteropexy are: requirement of mesh and treatment of incontinence. Arthur and Savage were the first to describe attachment of prolapsed uterus to sacrum.

As shown in the Table 11, the sample size for women who underwent abdominal sacrohysteropexy in this study was comparable with study by Tahir et al. ${ }^{12,13}$ The mean age for women who underwent abdominal sacrohysteropexy in this study was 25.6 years as compared to studies by Tahir et al and Barranger et al, where the mean ages were 30 years and 35.7 years respectively. ${ }^{14}$ The mean parity in this study was $66.67 \%$ among multipara and $33.34 \%$ among nullipara. Tahir et al had mean parity of $83.3 \%$ among multipara and $16.6 \%$ among nullipara. ${ }^{13}$ Most of the women in this study who underwent abdominal sacrohysteropexy had $3^{\text {rd }}$ degree prolapse which was comparable to studies by Barranger et al and Demirci and Leron. ${ }^{15}$

The mean age for comparison and grade of prolapse being the same (approx.), low parity and early follow up after abdominal sacrohysteropexy fetched us $100 \%$ success rate as compared to other studies. Study did not experience any case of recurrence post-operatively, while all other studies had recurrences of prolapse after surgery. Barranger et al has also mentioned about 1 mesh rupture and 4 intra-op complications encountered in their study. ${ }^{14}$

Follow-up period of this study was 6-7 months which was less as compared to studies by Tahir et al, Barranger et al and Demirci and Leron where the follow up period was 12 months, 44.5 months and 25 months respectively. $^{13-15}$

The limitations of this study were a small sample size as most of the women with symptomatic prolapse were not willing to participate in the study, lack of long-term follow-up due to non-compliance of women, limited data available regarding longevity of procedure.

\section{CONCLUSION}

Young women considering treatment of pelvic organ prolapse usually desire for uterine preservation. So, nonsurgical management of prolapse - remains first line of management. Amongst surgical management, abdominal sacrohysteropexy is preferred as it is safe, effective, maintains durable anatomic restoration of normal vaginal axis, preserves sexual function without painful or rigid vaginal scarring, has minimal complications with excellent success rate of $85 \%$.

\section{ACKNOWLEDGMENTS}

Authors would like to thank the residents for their cooperation, staff members and head of department of obstetrics and gynecology for their help in conduction of the study.

Funding: No funding sources

Conflict of interest: None declared

Ethical approval: Not required

\section{REFERENCES}

1. Downing KT. Uterine prolapse: from antiquity to today. Obstet Gynecol Inter. 2012:Article ID 649459:9.

2. Thakare PY, Mahale AR. Assessment of prolapse by Pelvic Organ Prolapse Quantification (POP-Q) System. Indian J Basic Appl Med Res. June 2014:3(3):324-30.

3. ACOG (American College of Obstetrics and Gynaecology) practice bulletin - clinical management guidelines for obstetrician and gynecologist. Obstet Gynaecol. 2007;110(3):717-27.

4. Olsen AL, Smith VG, Bergstrom JO, Colling JC, Clark AL. Epidemiology of surgically managed pelvic organ prolapse and urinary incontinence. Obstet Gynecol. 1997;89:501-6.

5. Vandana D, Shakun S, Rachna C, Manisha S, Singh M. Association between the standardized pelvic organ prolapse quantification system (POP-Q) and Shaw's system of classification for pelvic organ prolapse. Indian $\mathrm{J}$ Obstet Gynecol Res. 2017;4(2):166-70.

6. Samuelsson EC, Arne Victor FT, Tibblin G, Svardsudd KF. Signs of genital prolapse in a Swedish population of women 20 to 59 years of age and possible related factors. Am J Obstet Gynecol. 1999;180(2):299-305.

7. Jackson SR, Avery NC, Tarlton JF, Eckford SD, Abrams P, Bailey AJ. Changes in metabolism of collagen in genitourinary prolapse. Lancet. 1996;34:1658-61.

8. Versi E, Cardozo L, Brincat M, Cooper D, Montgomery J, Studd J. Correlation of uretral physiology and skin collagen in postmenopausal women. Br J Obstet Gynaecol. 1988;95:147-52.

9. Pandit SN. Introduction. In: Pandit SN, eds. Pelvic Organ Prolapse. $1^{\text {st }}$ Ed. India, National; 2013:1-2.

10. Campeau L, Gorbachinsky I, Badlani GH, Andersson K. Pelvic floor disorders: linking genetic risk factors to biochemical changes. BJU Int. 2011;108:1240-7.

11. Bortolini MA, Rizk DE. Genetics of pelvic organ prolapse: crossing the bridge between bench and bedside in urogynecologic research. Int Urogynecol J. 2011;22:1211-9.

12. Khan A, Jaleel R, Nasrullah FD. Sacrohysteropexy performed as uterus conserving surgery for pelvic organ prolapse: Review Case Files. Pak J Med Sci. 2016;32(5):1174-8.

13. Tahir S, Yasmin N, Kanwal S, Aleem M. Abdominal sacrohysteropexy in young women with uterovaginal prolapse. APMC. 2012;6(1):75-80. 
14. Barranger E, Fritel X, Pigne A. Abdominal sacrohysteropexy in young women with uterovaginal prolapse; long term follow up. Am J Obstet Gynecol. 2003;189:1245-50.

15. Demirci F, Ozdemir. Adominal sacrohysteropexy in young women with uterovaginal prolapse: result of 20 cases. J Reprod Med. 2006;51:539-43.
Cite this article as: Bhalerao AV, Duddalwar VA. Abdominal sacrohysteropexy versus vaginal hysterectomy for pelvic organ prolapse in young women. Int J Reprod Contracept Obstet Gynecol 2020;9:1434-41. 